\title{
Microsystèmes communicants : modélisation, fabrication et mesures
}

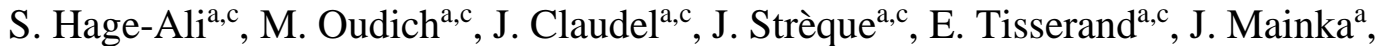 \\ D. Rouxel ${ }^{\mathrm{a}, \mathrm{c}}$, C. Floer $^{\mathrm{c}}$, O. Elmazria ${ }^{\mathrm{b}, \mathrm{c}}$, M. Hehn ${ }^{\mathrm{b}, \mathrm{c}}$, F. Weinachter ${ }^{\mathrm{a}}$, S. Weber ${ }^{\mathrm{a}, \mathrm{c}}$ \\ ${ }^{a}$ Faculté des Sciences et Technologies, Université de Lorraine, 54506 Vandœuvre-Lès- \\ Nancy, France Nancy 54000, France \\ b Polytech'Nancy, Université de Lorraine 2, rue Jean Lamour | 54519 Vandœuvre-Lès- \\ Nancy Cedex \\ c Institut Jean Lamour, UMR 7198, Université de Lorraine - CNRS, Nancy 54000, France \\ Contact email : $\underline{\text { sami.hage-ali@ univ-lorraine.fr, mourad.oudich@univ-lorraine.fr }}$
}

Nous présentons la mise en place de travaux pratiques et projets pédagogiques portant sur l'initiation à la modélisation, la fabrication et le test de microsystèmes communicants. Cette formation s'adresse aux étudiants de la licence 3 SPI et du Master EEA, parcours Capteur Intelligents Micro et nanotechnologie (CIM) à l'Université de Lorraine. Les travaux pratiques s'appuient d'une part sur de la modélisation par la méthode des éléments finis (FEM), et sur fabrication et la caractérisation des micro-dispositifs, notamment grâce à des ressources de l'Institut Jean Lamour à Nancy. L'exemple d'une étude de cas autour d'un capteur sans fil intégrant ces ressources est détaillé.

\section{Introduction}

Actuellement les micro-capteurs/actionneurs communicants couvrent un vaste champ d'applications bien connues incluant la surveillance, le contrôle interactif, la géolocalisation, les systèmes automatisés dans les domaines de l'automobile et l'aéronautique, sans oublier le domaine médical. On assiste à un développement sans précédent de ces dispositifs pour la détection et la commande avec une forte de compétences relatives à leur conception et mise en œuvre dans le marché de l'emploi. Il est donc important de doter nos étudiants des compétences nécessaires pour la conception, la fabrication et le test de ces microsystèmes, de manière à pouvoir appréhender leurs développements et innover dans le domaine.

Cette communication présente les travaux pratiques et les projets pédagogiques qui ont été mis en place pour initier et former les étudiants à la modélisation, la fabrication et le test des micro-dispositifs communicants au niveau de la licence 3 Sciences pour l'Ingénieur (SPI) et du Master EEA, parcours Capteur Intelligents Micro et nanotechnologie (CIM), au sein du Département d'Electronique et d'Electrotechnique (DEE) de la faculté de Sciences et Technologie de l'Université de Lorraine. Ces formations ont été fortement appuyées par le CNFM sur deux projets intitulés « Simulation des microsystèmes et des dispositifs RF » en 2016 et « Microcapteurs communicants » en 2017. 


\section{Les travaux pratiques mis en place : matériel et objectifs pédagogiques}

\section{Modélisation des microsystèmes}

L'ingénierie des systèmes complexes nécessite des moyens de conception et d'optimisation qui se base actuellement sur la modélisation numérique. Grâce aux machines de calculs qui deviennent de plus en plus puissantes, un système de détection ou d'actionnement peut être conçu, modélisé, testé et optimisé virtuellement avant sa fabrication et caractérisation, ce qui permet une grande économie en temps et en coût.

Le logiciel COMSOL Multiphysics a été choisi comme base commune de tous les enseignements incluant une composante de modélisation physique, et été acquis dans le cadre de notre projet 2016.

En licence SPI, la modélisation par la méthode des éléments finis avec pour objectif d'illustrer les concepts théoriques introduits en cours et de faire découvrir aux étudiants les phénomènes physiques (voir Fig. 1) et les spécificités du calcul numérique. Cela concernera plus particulièrement les phénomènes de transfert de chaleur, la mécanique et les ondes électromagnétiques.

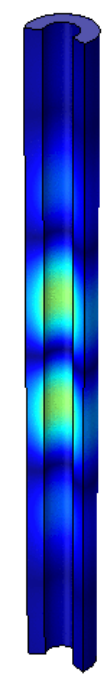

Fig.1. Simulation de la propagation d'une onde après réflexion sur une charge de type court-circuit, à l'aide du logiciel COMSOL Multiphysics. Illustration de la solution de l'équation des télécommunications.

Au niveau du Master EEA CIM, trois Unités d'Enseignement (UEs) spécifiques à la simulation des capteurs et antennes ont été créées avec comme objectifs :

- faire découvrir aux étudiants les couplages multi-physiques dans un système complexe,

- apprendre aux étudiants à entreprendre une démarche d'ingénierie de simulation numérique en s'appuyant sur un plan de travail incluant les étapes de conception, de modélisation et d'optimisation d'un système afin de répondre à un cahier de charges bien précis.

Micro-capteurs communicants : fabrication, caractérisation et traitement du signal

Notre projet CNFM 2017 nous a permis de renforcer significativement nos capacités pédagogiques dans trois sous-domaines, qui viennent supplémenter des ressources de microfabrication et caractérisation déjà présentes à la FST (ATELA) et à l'Institut Jean Lamour. 
a) La communication sans fil et l'impédancemétrie

Nous avons développé des TP illustrant les principes de fonctionnement des antennes et capteurs sans fil, ainsi que généralement des dispositifs dont l'impédance varie. Le matériel associé a concerné concerne l'achat de mini-analyseurs de réseaux (MiniVNATiny, miniradiosolutions) et de cartes «lock-in» (Analog Devices AD8302) pour l’impédancemétrie dynamique.

b) L'acquisition et le traitement du signal des capteurs

Cette partie concerne l'acquisition de matériel (DSPPic et MBED) et programmateurs associés pour l'acquisition et le traitement de signaux issus de capteurs commerciaux (gyroscopes/accéléromètres, capteurs de gaz, pression, magnétométrie Hall et GMR, ECG etc..). Le but ici est vraiment d'aller de la physique jusqu'au système.

c) Les micronanosystèmes

Nous avons pu renforcer les TP dans la salle «Nano» de l'Institut Jean Lamour, notamment l'AFM.

\section{Un exemple d'enseignement mis en place intégrant une grande partie de ces ressources pédagogiques : le module de master « Etude de cas : capteur de température sans fil souple à base d'un résonateur SAW : conception, réalisation, test »}

Le but de module «Etude de cas : capteur de température sans fil souple à base d'un résonateur SAW : conception, réalisation, test » est de consolider une grande partie des enseignements grâce à une étude cas complète. Il s'agit de totalement concevoir, réaliser et tester un capteur de température sans fil basé sur un résonateur à onde acoustique de surface. ( $c f$. figure 2). Cette étude est rendue possible dans un volume horaire limité par la relative simplicité de fabrication des capteurs SAW et des antennes en salle blanche (une étape de lithographie/séance de 4 h à chaque fois)

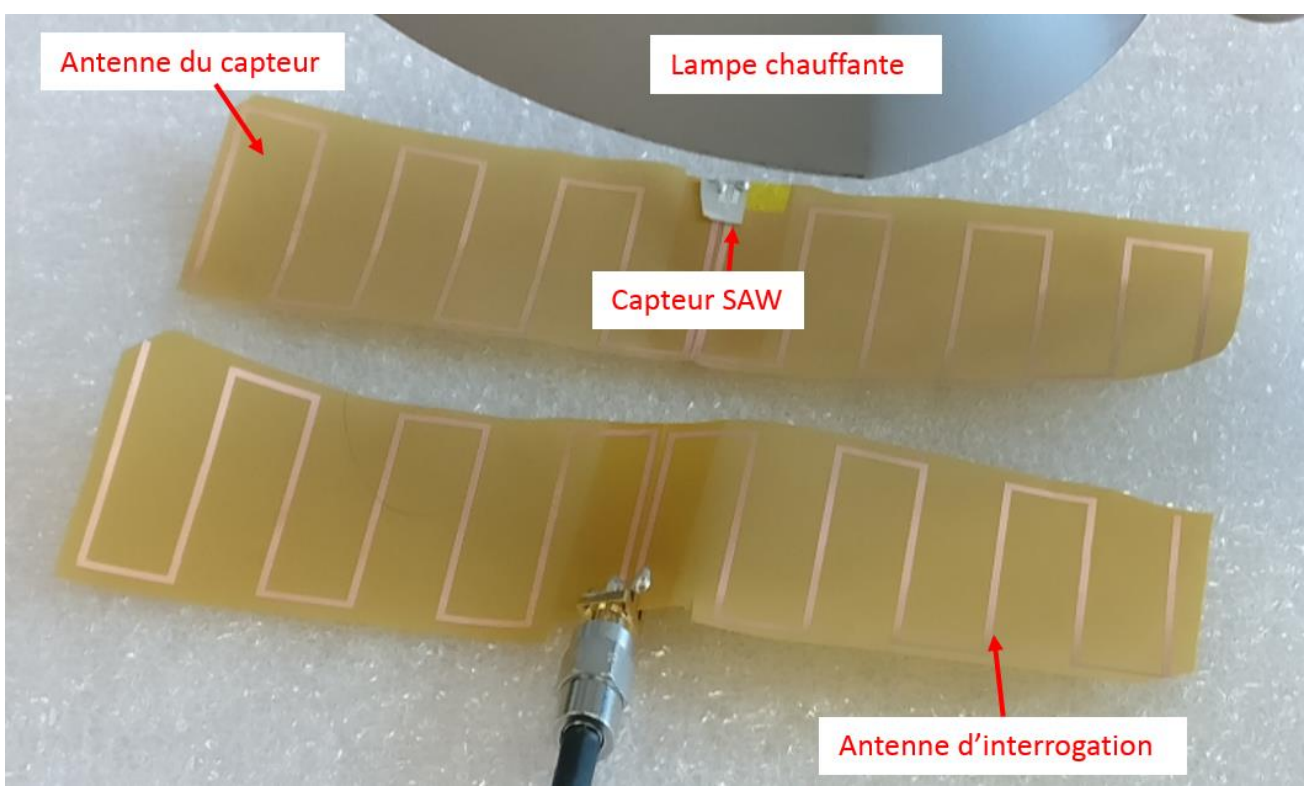

Fig.2. Capteur SAW sans fil à $390 \mathrm{MHz}$ réalisé par les élèves. 
Cette étude se déroule en plusieurs étapes :

- le point de départ de l'enseignement est une étude bibliographique sur ce type de capteur pour familiariser l'élève avec la technologie,

- design de l'antenne : l'élève doit ensuite concevoir une antenne à $390 \mathrm{MHz}$. La fabrication imposant une surface maximale pour l'antenne, il est redirigé vers un design en méandres qu'il doit modéliser et optimiser grâce au module RF de COMSOL. Le design d'antennes sur logiciel aura été abordé précédemment dans le module «Hyperfréquences et capteurs sans fil ». Cette partie dure 2 séances,

- fabrication de l'antenne en salle blanche: le design (différent) de chaque groupe d'élève est fabriqué en salle blanche, grâce à l'outil de lithographie sans masque développé par la société SmartForceTechnologies, sur un feuillet de Kapton métallisé. Cette technique utilise une matrice HD LCD pour définir un motif arbitraire en lumière bleue (g-line 436nm), pixel par pixel, à travers une optique focalisatrice ( $c f$. figure 3),

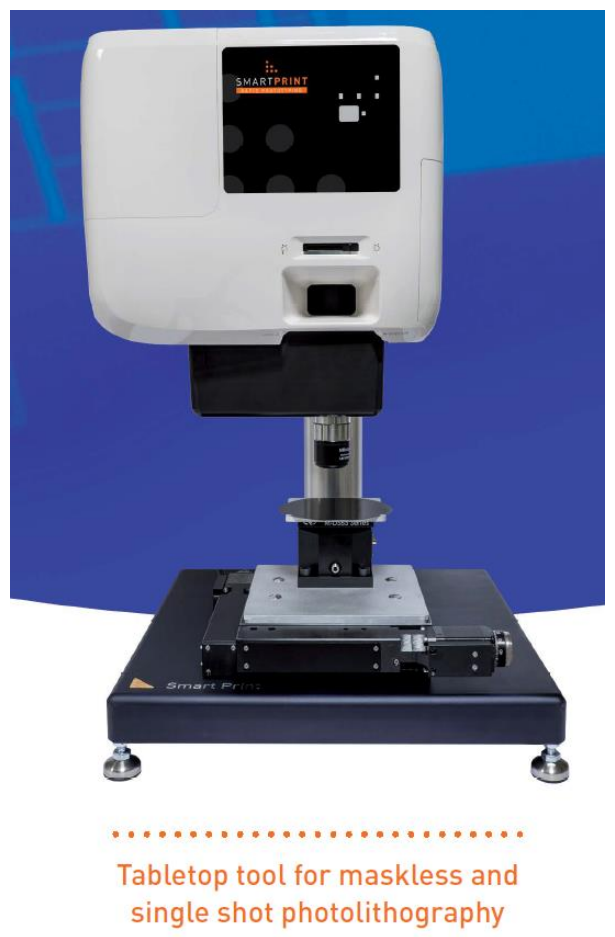

Fig.3. Système de photolithographie sans masque, SmartForceTechnologies, Grenoble, France.

- simulation du capteur SAW : le capteur SAW est modélisé sur le logiciel COMSOL Multiphysics (module «Structural mechanics » grâce à un modèle 2D périodique. La dépendance en température des paramètres élastiques est implémentée de manière à estimer la dépendance en fréquence du résonateur. Cette sous-partie est abordée de manière préalable dans le module « Initiation à la modélisation physique »,

- fabrication du capteur SAW en salle blanche : une autre séance est consacrée à la fabrication du capteur SAW en salle blanche, par pulvérisation cathodique / lithographie et gravure chimique d'une couche d'aluminium sur substrat niobate de lithium. Les bases des micro-nanotechnologies et la microfabrication salle blanche sont préalablement abordées dans le module «Technologies et caractérisation des micro-nano-systèmes ». Le fort investissement de l'Institut Jean Lamour et de son centre de compétence CC-Minalor (Centrale Lorraine de Micro-Nanotechnologies) 
dans les formations de niveau master permet l'accès des étudiants à une ressource de haut niveau.

- mesure des capteurs et antennes : après fabrication les capteurs SAW sont ensuite mesurés sur un analyseur de réseau présent dans la salle «Nanotechnologies » de 1'Institut Jean Lamour, une salle dédiée à l'enseignement de techniques avancées (outre le VNA, des TP d'AFM, STM, MEB sont présents). De leur côté, les antennes sont mesurées sur les miniVNA de la société Miniradiosolutions. Extrêmement faciles de prise en main et de coup très modique ( $<400$ euros), ces miniVNA peuvent équiper de nombreux postes dans une salle. Une comparaison avec les modélisations COMSOL est effectuée et discutée,

- mesure du capteur sans fil : enfin les capteurs SAW et antennes fabriquées par les élèves sont connectés et testés sans fil avec une antenne d'interrogation, sous lampe chauffante (voir Fig. 2), et le TCF (Temperature Coefficient of Frequency) mesuré.

En somme, il a été possible d'intégrer de nombreuses notions et matériels, pour la plupart introduits au préalable, au sein d'une seule étude «capteurs » complète. Cette transversalité, ainsi que la possibilité pour l'étudiant de concevoir, créer et tester un objet de toute pièces a fait l'objet d'un retour très positif.

\section{IV.Conclusion}

Nous avons pu présenter dans cette communication différents efforts de développement de travaux pratiques innovants orientés vers les microcapteurs communicants au niveau licence et master, à la Faculté de Sciences et Technologies de l'Université de Lorraine, rendus possibles par le soutien du GIP-CNFM.

\section{Remerciements}

Nous remercions ici tous les personnels d'ATELA (FST), notamment Jérôme Kadok et du centre de compétence MINALOR de l'Institut Jean Lamour (Gwladys Lengaigne, Laurent Badie, Stefan McMurtry, Carlos Rojas Sanchez). Sans leur investissement, ces travaux pratiques ne pourraient pas avoir lieu. Nous remercions également, le GIP-CNFM [1] et le projet FINMINA [2], ainsi que le directeur du Pôle CNFM MIGREST, Luc Hébrard. Enfin, nous remercions la région Grand Est, le FEDER et l’Université de Lorraine qui ont cofinancé ces TP.

\section{Références}

1. GIP-CNFM: Groupement d'Intérêt Public - Coordination Nationale pour la formation en Microélectronique et en nanotechnologies. Website: http://wwww.cnfm.fr (Accès juillet 2018)

2. IDEFI-FINMINA : Initiative d'Excellence - Formation Innovante en MIcroélectronique et Nanotechnologies, ANR-11-IDFI-0017. Website: http://www.cnfm.fr/VersionFrancaise/actualites/ FINMINA.htm (Accès juillet 2018) 